dyes are relatively good inhibitors of in vitro hæmatincatalysed oxidation of unsaturated fat.

Since the hæmatin compounds are the predominant catalysts occurring in animal tissues ${ }^{3}$ for the oxidation of unsaturated fats, the ability of these redox dyes to replace vitamin $\mathbf{E}$ in the diet of rats is probably a function of their antioxygenic properties, and more specifically, of their inhibitory effect on the hæmatincatalysed oxidation of unsaturated fats.

Hæmatin is capable of chemical combination with many nitrogenous bases, through its iron atom, to form hemichromes. It is probable that these redox dyes, all of which contain basic nitrogen atoms, react with hæmatin to form hemichromes which are not catalytic. The direct interaction of hæmatin with methylene blue is also indicated by the fact that the absorption spectrum of a mixture of methylene blue and hæmatin is considerably different from that of the summation of the absorption spectra of the individual compounds.

Besides direct reaction of these redox dyes with hæmatin, two other mechanisms might be operative in vivo. The oxidation-reduction potential of most animal tissues and organs is sufficiently negative in comparison to that of the redox dyes to account for the existence of the leuco form in vivo. These leuco dyes might function by inhibiting the oxidation of unsaturated fats by terminating the free-radical chain-reaction. Alternatively, the dyes might serve to regenerate $\alpha$-tocopherol from a reversible oxidation product, like $\alpha$-tocopheroxide, and thus allow the re-use of otherwise subminimal amounts of vitamin $\mathrm{E}$.

V. P. Mater

A. L. TAPPEL

Department of Food Technology,

University of California, Davis, California. Jan. 5.

${ }^{1}$ Moore, T., Sharman, I. M., and Ward, R. J., Biochem. J., 53, $\operatorname{xxx}$ (1953);

Tappel, A. L., Arch. Biochem. Biophys., 50, 473 (1954).

sappel, A. L., Food Res., 18, 104 (1953).

\section{An Optically Active Spirocyclic Phosphonium Salt}

THE tetrahedral disposition of the 4-covalent phosphorus atom has been confirmed by the optical resolution of $(a)$ a tertiary phosphine oxide, of type $a b c \mathrm{PO}$, by Meisenheimer et al.1, (b) a tertiary phosphine sulphide, abcPS, by Davies and Mann', and (c) a quaternary phosphonium salt, [abcdP]I, by Holliman and Mann ${ }^{3}$. No spirocyclic phosphonium salts, in which the two ring systems consist solely of carbon atoms apart from the phosphorus atom which is necessarily common to both, have, however, hitherto been recorded.

The comparatively ready synthetic methods by which analogous spirocyclic arsonium salts ${ }^{4}$ have been prepared cannot be successfully applied to the phosphonium salts. By a much longer route, however, we have synthesized $d l$-P-spiro-bis-1 $: 2: 3: 4$-tetrahydrophosphinolinium iodide $(\mathrm{I})$, melting point $294-$ $295^{\circ}$. The optical resolution of this dissymmetric cation has been achieved by the fractional crystallization of its $l$-menthoxyacetate, and we have thus isolated the $d$-iodide, melting point $246-248^{\circ},[M]_{D}+$ $66^{\circ}$, and the $l$-iodide, melting point $246-248^{\circ}$, $[M]_{D}-65^{\circ}$, both rotations determined in chloroform

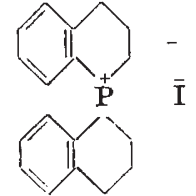

(I)

solution. These rotations showed no change after the solution had been set aside at room temperature for four days.

\section{University Chemical Laboratories,}

F. G. MANN

F. A. HaRT

Cambridge.

April 2.

1 Ber., 44, 356 (1911); Annalen, 449, 224 (1926).

J. Chem. Soc., 276 (1944).

J. Chem. Soc., 1634 (1947).

- Holliman and Mann $J$. Chem. Soc., 45 (1945).

\section{Correction of 'Crude-Fibre' Estimation for the Effect of Altitude}

Is the estimation of crude fibre at altitudes appreciably above sea-level, it is necessary to correct for lowered boiling points of reagents and consequent reduced speeds of reaction. When Todd ${ }^{3}$ estimated crude fibre in ten samples of plant materials at sealevel and at 6,200 $\mathrm{ft}$., he calculated the percentage error involved in each instance and proposed three ways of correcting, namely : (1) by increasing the duration of the boiling; (2) by increasing the concentration of acid and alkali ; (3) by carrying out the estimation by the standard procedure and applying a correction factor.

An examination of the first proposal was made by me in Nairobi, at an altitude of 5,700 ft. Twelve samples of plant material of varying fibre content were digested with $1 \cdot 25$ per cent acid and with 1.25 per cent alkali for periods of $15,30,45,60,90$ and $180 \mathrm{~min}$. Results showed that there is a highly significant inverse relationship between the logarithm of the time allowed for digestion and the resulting crude-fibre fraction of the material. In Nairobi, the reagents boil at $94 \cdot 3^{\circ} \mathrm{C}$., and a digestion period of $45 \mathrm{~min}$. gives crude fibre values which do not differ significantly from those obtained by the conventional $30 \mathrm{~min}$. digestion at sea-level: a calculated digestion period of $45 \cdot 6 \mathrm{~min}$. appears to give optimum correlation.

The following equation has been evolved to correct for lowered boiling points at higher altitudes by increasing the duration of the digestion period :

time of digestion $(\min )=.30 \times 10^{\left(1000-t^{0}\right) 0.032}$

where $t^{\circ} \mathrm{C}$. is the boiling point of the reagents.

Alternatively, the regression equation to correct the conventional 30 min. digestion value will be: crude fibre $($ corrected $)=$ crude fibre $\left(30\right.$ min. at $\left.t^{\circ}\right) \times$ $\left[1-\left(100^{\circ}-t^{\circ}\right) 0.0102\right]$

H. W. Dougall

School of Agriculture,

King's College,

University of Durham.

Feb. 2.

1 Todd, J. R., Nature, 168, 76 (1951). 